\title{
Geometrid larvae of the Alpi Marittime Natural Park (district of Valdieri, Cuneo, Italy), with descriptions of the larvae of two Gnophini Pierce, 1914 (Insecta: Lepidoptera: Geometridae)
}

\author{
Gareth Edward KING \\ Departamento de Biología (Zoología), \\ Universidad Autónoma de Madrid, 28069 Cantoblanco, Madrid (Spain) \\ sterrhinae@gmail.com \\ Félix Javier GONZÁLEZ-ESTÉBANEZ \\ Departamento de Biodiversidad y Gestión Ambiental, \\ Universidad de León, 24071 León (Spain) \\ fjgonzest@gmail.com
}

Published on 31 December 2015

urn:Isid:zoobank.org:pub:36697C66-C6FF-4FC0-BD97-303C937A0BEF

King G. E. \& González-Estébanez F. J. 2015. - Geometrid larvae of the Alpi Marittime Natural Park (district of Valdieri, Cuneo, Italy), with descriptions of the larvae of two Gnophini Pierce, 1914 (Insecta: Lepidoptera: Geometridae), in Daugeron C., Deharveng L., Isaia M., Villemant C. \& Judson M. (eds), Mercantour/Alpi Marittime All Taxa Biodiversity Inventory. Zoosystema 37 (4): 621-631. http://dx.doi.org/10.5252/z2015n4a8

$$
\begin{array}{r}
\text { KEY WORDS } \\
\text { Insecta, } \\
\text { Lepidoptera, } \\
\text { Geometridae, } \\
\text { Gnophini, } \\
\text { Italy, } \\
\text { Maritime Alps, } \\
\text { larvae, } \\
\text { morphology, } \\
\text { chaetotaxy. }
\end{array}
$$

MOTS CLÉS

Insecta,

Lepidoptera,

Geometridae,

Gnophini,

Alpes Maritalie

ritimes,

larve,
morphologie,

orphologie,

\section{ABSTRACT}

Examination of 14 plant families in the Alpi Marittime Alps Natural Park (Valdieri, Italy) resulted in the collection of 103 larvae of 28 geometrid taxa; these belong to three subfamilies, with Ennominae Duponchel, 1845 being the most representative $(13$ taxa $=46.4 \%)$. The final instar $(\mathrm{L} 5)$ of two taxa in the tribe Gnophini Pierce, 1914, Gnophos furvata meridionalis Wehrli, 1924 and Charissa pullata ([Denis \& Schiffermüller], 1775) is described, including its chaetotaxy. Biological data and observations are provided for all taxa.

\section{RÉSUMÉ}

Les larves de Geometridae collectées dans le Parc naturel des Alpi Marittime (district Valdieri, Cuneo, Italie), avec la description des larves de deux espèces de Gnophini Pierce, 1914 (Insecta: Lepidoptera: Geometridae). L'examen de 14 familles de plantes a permis de récolter 103 juvéniles appartenant à 28 espèces de Geometridae Leach, 1815 de trois sous-familles différentes. Les Ennominae Duponchel, 1845 sont les plus représentées, avec 13 taxons (46,4\%). Le dernier stade larvaire (L5) de deux espèces de la tribu des Gnophini Pierce, 1914 : Gnophos furvata meridionalis Wehrli, 1924 et Charissa pullata ([Denis \& Schiffermüller], 1775) est décrit, y compris pour la chétotaxie. Des données et observations biologiques sont proposées pour tous les taxons. 
Table 1. - Valdieri district (Italian: capoluogo) locality data.

\begin{tabular}{|c|c|c|}
\hline Locality & Altitude & Coordinates \\
\hline Entracque & $845-908 \mathrm{~m}$ & $\begin{array}{r}44^{\circ} 14^{\prime} 53.0^{\prime \prime} \mathrm{N} \\
7^{\circ} 22^{\prime} 57.2^{\prime \prime} \mathrm{E}\end{array}$ \\
\hline Lago della Rovina & $1533-1543 \mathrm{~m}$ & $\begin{array}{r}44^{\circ} 10^{\prime} 55.2{ }^{\prime \prime} \mathrm{N} \\
7^{\circ} 20^{\prime} 39.1 \text { 'E }\end{array}$ \\
\hline Ponte della Vagliotta & $1146-1260 \mathrm{~m}$ & $\begin{array}{l}44^{\circ} 13^{\prime} 07.0^{\prime \prime} \mathrm{N} \\
7^{\circ} 17^{\prime} 08.2^{\prime \prime} \mathrm{E}\end{array}$ \\
\hline $\begin{array}{l}\text { Riserva Naturale Ginepro } \\
\text { di Fenecia }\end{array}$ & $776-1031 \mathrm{~m}$ & $\begin{array}{r}44^{\circ} 28^{\prime} 17.7{ }^{\prime \prime} \mathrm{N} \\
7^{\circ} 39^{\prime} 68.33^{\prime \prime} \mathrm{E}\end{array}$ \\
\hline San Lorenzo & $827-885 \mathrm{~m}$ & $\begin{array}{r}44^{\circ} 16^{\prime} 54.1{ }^{\prime \prime N} \\
7^{\circ} 23^{\prime} 56.11^{\prime \prime} \mathrm{E}\end{array}$ \\
\hline Sant'Anna di Valdieri & $924-1.022$ m & $\begin{array}{r}44^{\circ} 15^{\prime} 00.4^{\prime \prime} \mathrm{N} \\
7^{\circ} 20^{\prime} 16.33^{\prime \prime} \mathrm{E}\end{array}$ \\
\hline Ubac di Rugeral & $1319 \mathrm{~m}$ & $\begin{array}{r}44^{\circ} 12^{\prime} 47.3^{\prime \prime} \mathrm{N} \\
7^{\circ} 16^{\prime} 41.3^{\prime \prime} \mathrm{E}\end{array}$ \\
\hline Vallone de Chistofort & $1291-1313 \mathrm{~m}$ & $\begin{array}{r}44^{\circ} 11^{\prime} 37.99^{\prime \prime} \mathrm{N} \\
7^{\circ} 21^{\prime} 30.8^{\prime \prime} \mathrm{E}\end{array}$ \\
\hline
\end{tabular}

\section{INTRODUCTION}

In the context of a faunistically important European mountain belt such as the Alps, more specifically the Italian Alps, the bibliography dealing with geometrid larvae and their biology is scant: Carrara (1926-1928); Sannino et al. (1995); Sannino \& Espinosa (1999, 2002); Huemer \& Morandini (2006) and Flamigni et al. (2007). Baldizzone et al. (2005), based on the results of a six-year field campaign (using light traps), listed 220 Geometrid taxa (including bibliographical references) for the Alpi Marittime (117 species in the Larentiinae [53.2\%]; 56 in the Ennominae Duponchel, 1845 [25.5\%]), which represented approximately one-third of the Italian fauna (Parenzan \& Porcelli 2006). Huemer (1998) listed 17 Geometrid species as being endemic to the Alps, representing $1.9 \%$ of the European Geometrid fauna, while Ozenda (1966) recognized the biogeographical importance of the (south) western Alps with sub-Mediterranean, Alpine and Central Europe elements being represented, whilst at the same time having connections with the SE and Dinaric Alps.

Morphological data (including studies of the chaetotaxy) of Geometrid larvae is not at the same level of availability as for the Noctuoidea Latreille, 1809 (Beck 1960; Ahola \& Silvonen 2005). Synapomorphies of Geometrid larvae include prolegs on abdominal segments (urites) A6 and A10 (Singh 1951), with exceptions in certain subfamilies (e.g., Archiearinae Fletcher, 1953), an additional characteristic is the presence of an extra seta amongst the lateral setae group (L1-L4) (these are primary setae present on larval eclosion from the egg) (Dugdale 1961); secondary setae (after the first two instars), if present, are found on the abdominal urites (A1-A10), with the presence of numerous microscopic secondary setae laterally, posteriorly and in the cephalic region (Singh 1951). The location of various protuberances or cuticular processes can be observed in the subfamily Ennominae (Bocaz et al. 2003) as, for example, in the tribe Gnophini Pierce, 1914 (Trusch \& Erlacher 2001; Ebert et al. 2003). Another synapomorphy is the presence of four setae on the anterior surface of the A6 prolegs, which can be considerably more (Young 2008). Occasionally the abdominal prolegs (A6) can give the impression of being underneath the A7 segment (Forbes 1910; Singh 1951). The anal prolegs (A10) have seven setae, as well as three placed posteriorly, although the number of setae is not a constant feature and there can be more than nine (Singh 1951). Ahola \& Silvonen (2005) name the setae on the anal shield (of Noctuoidea larvae), but not on the anal proleg (A10). With reference to the thoracic region, the lateral setae (L1-L3) are bisetose or trisetose; spiracle present on segment T1 (absent on T2 and T3); seta SV2 absent on A1 (Dugdale 1961; Stehr 1987).

Papers that have dealt with the larval chaetotaxy of Ennominae include Singh (1951), Dugdale (1961), Sato (1984), Wagner et al. (2003) and Young (2008). Here we give for the first time the L5 larval chaetotaxy of two species in the Gnophini.

This present paper aims to provide biological data on the geometrid larvae that were collected during a series of field trips that took place in the vicinity of Valdieri (Table 1). These data include food-plants, parasitoids (Diptera; Hymenoptera) and larval phenology. Concerning the former, we have excluded as "food-plants" vegetation on which the larvae were merely resting on, since larvae often perch away from the food-plant in order to avoid attack by parasitoids (Diptera, Hymenoptera) (Turlings et al. 2003), as well as plants that might offer structural support only (protection from the weather or as an overwintering [or aestivating] "roost"; Fielding \& Coulson 1995).

\section{MATERIAL AND METHODS}

\section{STUDY AREA}

The French-Italian western Alps (Maritime Alps) reach down SW from Léman (Switzerland) to the Gulf of Genoa. They have a characteristic oceanic influence, an annual precipitation of $1200-1600 \mathrm{~mm}$, with a continentality index of $30-40^{\circ} \mathrm{C}$ (Blanchard 1956), being a continuation of the Pyrénées orientales in the west and the Apennines and the Balkans to the east (Barbero et al. 1971). Barbero (1981) includes the following plant associations in the northern Mediterranean mountains systems (of which the Alps form part): Querco-Fagetea, Vaccinio-Piceetea, Elyno-Seslerietea, Caricetea curvuleae, Festuco-Seslerieta, Pino-Juniperetea, in addition to Quercetea pubescentis. Barbero (1979) emphasized the increasing importance of Mediterranean botanical elements on the Italian side, even though summer drought conditions do not exceed a month (Aschmann 1984), with central European botanical elements still playing an important role (Barbero et al. 1971).

An important aspect of the methodology used was that of searching for larvae at ground level in order to include the Sterrhinae Meyrick, 1892, which are overwhelmingly nonarboreal (except the Cosymbini Prout, 1911), and include those taxa that use senescent leaves as a source of food, as is the case with the genus Idaea Treitschke, 1825 (Heitzman 1973; Covell 1983; Ebert \& Steiner 2001; Hausmann 2004). 


\section{COLLECTING AND IDENTIFICATION}

Larvae were collected according to the methods outlined in King \& Viejo Montesinos (2010); this involved searching for larvae at the base of possible food-plants, as well as examining leaf litter and scraping at the soil underneath prospective plants. Lifting nearby stones that were exposed or under low plants, was also undertaken. Plants inspected included labiates, polygonales, scrophulaceous plants, as well as grasses (Poaceae Barnhart) and other low plants, such as Viola L. (Violaceae Batsch), Hypericum L. (Asteraceae) and Epilobium Dill. ex L. (Onagraceae Juss.). Larvae were also beaten (using a stout branch) from the lower branches of shrubs or trees, including Salix caprea, Salix sp., Prunus spinosa, Betula alba, Corylus avellana, Amelanchia ovalis, Quercus sp., Crataegus sp., Pyrus communis, Acer sp., Ligustrum sp., Juniperus phoenica and Rosa canina, into a beating tray (Winter 2000), with specimens being collected from the debris accumulated in the tray. Larvae so collected were put into plastic vials with appropriate data included with the specimen: taxon (if known), larval instar (based on size, colouration or other features), plant in proximity and date.

Larvae were then taken back to the laboratory and reared to imago in order that a secure identification could be made. In captivity, larvae were maintained in plastic boxes with the appropriate food-plant, attended to daily and data kept as to pupation date, date of change to pupa, date of emergence of imago, etc. When parasitoid attacks were noted, data would include date of parasitoid activity (exit from larval host, whether cocoon constructed, date of emergence of dipteran or hymenopteran parasitoid) (Shaw 1997). In cases where larvae perished in captivity, they were preserved in $70 \%$ ethanol and stored as part of a (temporary) collection at the UAM.

Sample size was small for obtaining details on chaetotaxy: one mature larva (L5) of Gnophos furvata meridionalis Wehrli, 1924, and two larvae (L5) of Charissa pullata ([Denis \& Schiffermüller], 1775). Future studies should incorporate chaetotaxic studies of the L1 larvae ab ovo ex female in order to study the positioning of the primary setae. The present paper is only intended to provide a preliminary account of these previously unknown ennomine larvae.

Field work was performed on the following dates: 30 .VIII3.IX.2008; 4-7.VII.2009; 1-10.VIII.2009; 1-5.V.2010; 7-16. VIII.2010; 27-30.V.2011; 30.VIII-4.IX.2011.

Terminology follows Hinton (1946); Dugdale (1961); and Hassenfuss (1963).

Plants to species level (includes literature records) are mentioned in the Appendix.

\section{ABBREVIATIONS}

\section{Abbreviation used in the figures}

L1-L5 larval instars.

$\begin{array}{ll}\begin{array}{ll}\text { Collectors, } \\ \text { coll. }\end{array} & \text { collerminators and private collections } \\ \text { FJGE } & \text { Félix Javier González Estébanez; } \\ \text { GEK } & \text { Gareth Edward King; } \\ \text { MRS } & \text { Mark R. Shaw. }\end{array}$

Institutions

MNCN Museo Nacional de Ciencias Naturales, Madrid;

MRSN Museo Regionale di Scienze Naturali, Turin;

PNAM Parco Naturale Alpi Marittime;

RMS Royal Museum of Scotland, Edinburgh;

UAM Universidad Autónoma de Madrid.

\section{RESULTS}

Plants belonging to 14 families were examined. All were identified at least to genus, with 12 identified to species level (see Appendix). 103 larvae were collected: 93 larvae $(=90.1 \%)$ could be identified to species or sub-species; they represent 28 taxa, belonging to three subfamilies: Sterrhinae Meyrick, 1892 (eight species = 28.6\%), Larentiinae Duponchel, 1845 (seven species = 25\%) and Ennominae $(13$ taxa $=46.4 \%)$. The commonest species was Colotois pennaria (Linnaeus, 1761) $(17$ larvae $=16.5 \%)$, while 14 species were found only once $(=50 \%)$.

\section{Subfamily STERrHINAE Meyrick, 1892}

Idaea ochrata (Scopoli, 1763)

\section{Phalaena ochrata Scopoli, 1763: 227, fig. 567.}

MATERIAL EXAMINED. - Three larvae (L5) 27-28.V.2011: Sant'Anna di Valdieri (1.166 m) (emerged: 26.VI.2011: 2 ơ 1 9 (slide code 3742) GEK leg. et det.) found on the soil underneath mats of Rumex conglomeratus (King \& Viejo Montesinos 2012). Larva L4 28.V.2011 parasitized by Aleiodes crassipes (Thomson, 1892) (Braconidae: Rogadinae) (GEK leg., MRS det.; deposited RMS), a species not yet cited in Italy from the litterature (Villemant et al. 2015, this issue).

Notes ON FOOD-PLANT. - King \& Viejo Montesinos (2012) mention larvae of the subspecies I. ochrata albida (Zerny, 1936) being taken under mats of Rumex crispus L. and R. acetosa L. in Madrid (central Spain). The present record of larvae collected underneath mats of $R$. conglomeratus suggests that polygonales are important in the food-plant range of this taxon, although Waring (2004) indicates larvae being collected between small plants of Vicia cracca L. (Fabaceae) in southern England.

NOTES ON PARASITOID. - The L4 larva of this species attacked by Aleiodes crassipes is the first recorded case of a host in the Sterrhinae, although wasps of the genus Aleiodes Wesmael, 1838 do generally attack exposed "macrolepidoterid" larvae (Shaw \& Huddleston 1991; Falcó et al. 1997).

OTHER OBSERVATIONS. - Not previously documented from PNAM (Baldizzone et al. 2005).

\section{Idaea contiguaria (Hübner, 1799)}

Geometra contiguaria Hübner, 1799: pl. 20, fig. 105.

Material eXamined. - Larva (L5) 27.V.2011; Sant'Anna di Valdieri; (larva pupated 3.VI.2011, pupa formed 6.VI.2011, o' emerged 16.VI.2011; slide code 3740 GEK leg. et det., deposited MNCN) found on the soil underneath Silene sp. (Caryophyllaceae), which in turn was growing in-between Rumex conglomeratus (King \& Viejo Montesinos 2012). 
Notes ON FOOD-PLANT. - O Our data are insufficient to establish a food-plant association with Silene since the single larva taken was simply found underneath this plant.

Other OBSERVATIONS. - Previously recorded in PNAM (Baldizzone et al. 2005; King \& Viejo Montesinos 2012). It should be stated that Idaea larvae are notoriously difficult to collect in the wild (Covell 1983).

\section{Idaea aversata (Linnaeus, 1758)}

\section{Phalaena Geometra aversata Linnaeus, 1758: 526.}

MATERIAL EXAMINED. — Larva (L5) 28.V.2011 Sant'Anna di Valdieri (larva pupated: 3.VI.2011, o emerged 14.VI.2011; slide code 3739 GEK leg. et det., deposited MNCN) on soil underneath Rumex conglomeratus (King \& Viejo Montesinos 2012).

NOTES ON FOOD-PLANT. - The association with Rumex conglomeratus might suggest that this is a larval food-plant, but it could instead be that it is only acting as structural support.

OTHER OBSERVATIONS. - Previously recorded in the PNAM (Baldizzone et al. 2005; King \& Viejo Montesinos 2012).

\section{Idaea deversaria (Herrich-Schäffer, 1847)}

\section{Acidalia deversaria Herrich-Schäffer, 1847: 17.}

MATERIAL EXAMined. - Larva (L5), 29.V.2011 Vallone de Chistofort $(1291 \mathrm{~m})$ (larva pupated 6.VI.2011, pupa formed 8.VI.2011 \% emerged 18.VI.2011; slide code 3741, GEK leg. et det. deposited MNCN) on soil beneath Rumex conglomeratus (King \& Viejo Montesinos 2012).

NOTES ON FOOD-PLANT. - Not possible to establish with certainty the food-plant in the wild state.

Other observations. - Previously cited in the PNAM (Baldizzone et al. 2005; King \& Viejo Montesinos 2012).

Scopula incanata (Linnaeus, 1758)

\section{Phalaena Geometra incanata Linnaeus, 1758: 528.}

Material eXamined. - Larva (L5) 28.V.2011; Sant'Anna di Valdieri (981 m), between unidentified labiate species and Rumex conglomeratus (prepared to pupate 31.V.2011, pupa formed 2.VI.2011, O" emerged 11.VI.2011 (slide code 3743) GEK leg. et det. deposited $\mathrm{MNCN}$ ).

NOTES ON FOOD-PLANT. - Food-plant not known with certainty in the study area (Baldizzone et al. 2005). Larva taken in vicinity of Rumex conglomeratus, which agrees with general references to its food-plants as being polygonales (Ebert \& Steiner 2001; Hausmann 2004). Reared in captivity on Rumex crispus.

Other observations. - Previously cited in the PNAM (Baldizzone et al. 2005).

\section{Scopula marginepunctata \\ (Goeze, 1781)}

Phalaena Geometra marginepunctata Goeze, 1781: 385.

MATERIAL EXAMINED. - L5 Larva 6.IV.2009: Riserva Naturale Ginepro di Fenecia $(911-927 \mathrm{~m})$ in proximity to a clump of Thymus sp. (Labiatae) (O slide code 3482]; 11 L5 larvae 7.IV.2009, two specimens in proximity to Sedum sp. (Crassulariaceae), 9 (L5) specimens in proximity to Thymus sp. (\% slide code 3479; $20^{7} 0^{7}$ 3480, 3481 GEK leg. et det. deposited MNCN). All 12 specimens were collected exposed on open soil in full sunlight near to the aforementioned plants.

OTHER OBSERVATIONS. - Previously cited in the PNAM (Baldizzone et al. 2005). The finding of larvae on a bare substrate near to (but not on) possible food-plants and exposed to sunshine, concords with larvae of this species taken in central Spain (November to March) (King, pers. obs.), and to other species in the genus Scopula Schrank, 1802. This is possibly related to a "hide-escape" strategy adopted by the larvae, if female parasitoids are guided to their larval host by the chemicals released by leaves torn by phytophagous insects (Turlings et al. 2003).

\section{Rhodostrophia vibicaria (Clerck, 1759)}

Phalaena vibicaria Clerck, 1759: pl. 3, fig. 2.

Material eXAMINED. — Larva (L5) 1.V.2010; San Lorenzo (845 m) resting on Poaceae stem alongside Sedum sp. and Astragalus sp. (Leguminosae) (on emerged, GEK \& FJGE leg. et det. deposited MNCN); larva 2.V.2010 (L3) under mat of Lavandula sp. (Labiatae); Riserva Naturale Ginepro di Fenecia (did not survive); larva (L5) 28.V.2011; Ubac di Rugeral (1309 m); two larvae (L5); Sant'Anna di Valdieri; all three examples under Rumex scutatus (o" 3744; deposited MRSN); larva (L5) 29.V.2011 between labiate and Rumex scutatus; Lago della Rovina (1.546 m).

OTHER OBSERVATIONS. - Previously cited in the PNAM (Baldizzone et al. 2005). The mature larvae sit exposed on low plants during daylight hours (Ebert \& Steiner 2001), which is exactly how our material was taken. Rumex scutatus could well be the food-plant, since polygonales are mentioned generically in Hausmann (2004).

\section{Rhodometra sacraria (Linnaeus, 1767)}

Phalaena Geometra sacraria Linnaeus, 1767: 863.

Material eXAMINED. - Larva (L5) 2.IX.2008 in Oxyria digyna growing amongst scree; Riserva Naturale Ginepro di Fenecia (\% emerged; deposited MNCN); 2 larvae (L5) 2.IX.2009 in Rumex scutatus alongside river Gesso; San Lorenzo (emerged 18.VIII.2009; GEK leg. et det.; deposited MNCN).

NOTES ON FOOD-PLANT. - Although this divagant (Eitschberger et al. 1991) species is recognized as being oligophagous on polygonales (Gómez de Aizpúrua et al. 2006, amongst others), neither Oxyria digyna nor Rumex scutatus are documented as food-plants.

OTHER OBSERVATIONS. - Not previously documented in the PNAM (Baldizzone et al. 2005). The two occasions in late summer when the three larvae were collected is noteworthy in terms of the "corridor" function (Gallé et al. 1995) that the two localities would have served as the insect migrated northwards: an open scree slope alongside woodland and the banks of the river Gesso. 
Subfamily LARENTIINAE Duponchel, 1845

Catarhoe cuculata (Hufnagel, 1767)

Phalaena cuculata Hufnagel, 1767: 602.

MATERIAL EXAMinED. - Larva (L5), 6.VIII.2009, Entracque $(904 \mathrm{~m})$ wandering amongst herb layer in bright sunlight (foodplant not established); larva (L5) 15.VIII.2010, Sant'Anna di Valdieri (981 m) in Galium sylvaticum L., GEK leg. et det. (neither larva reared successfully).

OTHER OBSERVATIONS. - Previously cited in the PNAM (Baldizzone et al. 2005). Ebert \& Steiner (2001b) also mention the diurnal activity of this species' larva, especially as the first specimen was taken wandering in bright sunlight far from its assumed food-plants.

\section{Camptogramma bilineata}

(Linnaeus, 1758)

Phalaena Geometra bilineata Linnaeus, 1758: 525.

MATERIAL EXAMined. - Larva (L5), 2.V.2010, under low plants including Helianthemum (Cistaceae), Artemisia (Asteraceae), Astragalus and Lavandula, Riserva Naturale Ginepro di Fenecia (906 m); larva prepared to pupate 18.V.2010 (pupa perished) (deposited UAM); FJGE \& GEK leg., GEK det.

\section{Epirrhoe alternata (Müller, 1764)}

Phalaena Geometra alternata Müller, 1764: 51.

Material eXAmined. - Two larvae (L5): 9.VIII.2010 S. Anna di Valdieri $(981 \mathrm{~m})$ in Galium sylvaticum, GEK leg. et det. One larva parasitized by Protapanteles anchisiades (Nixon, 1986) (Braconidae: Microgastrinae); brood emerged: 21.VIII.2010; GEK leg. et MRS det. (deposited RMS); larva (L5): 3.IX.2011, Ponte della Vagliotta $(1.146-1.147 \mathrm{~m})$ in Galium sp. GEK leg. et det.

Notes ON PARASITOID. - Shaw \& Huddleston (1991) state that Protapanteles Ashmead, 1898 (Braconidae: Microgastrinae attack exposed "macrolepidopterid" larvae. Protapanteles anchisiades is an important parasitoid of Epirrita autumnata (Ruohomäki et al. 2013).

OTHER OBSERVATIONS. - Not previously documented from the PNAM (Baldizzone et al. 2005).

\section{Epirrhoe rivata (Hübner, 1813)}

Geometra rivata Hübner, 1813: 409.

MATERIAL EXAMINED. - Three larvae (L5): 7.VIII.2010, S. Anna di Valdieri on Galium sylvaticum, GEK leg. et det. (\% deposited MNCN).

\section{Epirrita dilutata (Denis \& Schiffermüller, 1775)}

Geometra dilutata Denis \& Schiffermüller, 1775: 109, pl. I a, b, frontispiece.

Material EXAmined. — Larva (L5): 3.V.2010, Sant'Anna di Valideri (924 m) on Corylus avellana (\% emerged 22.X.2010, slide code 467); leg. et det. FJGE, deposited in coll. FJGE.

\section{Aplocera praeformata}

(Hübner, 1826)

Geometra praeformata Hübner, 1826: pl. 103, figs 532, 533.

Material EXAMINED. — Larva (L5) resting on Sedum sp., 1.V.2010, San Lorenzo; GEK/FJGE leg., GEK det. (\% slide code 3677; deposited MNCN); two larvae (L5), 29.V.2011, Vallone de Chistofort $(1312 \mathrm{~m})$ under Rumex scutatus in proximity to Hieracium sp. (Asteraceae) (neither pupa survived; genitalia extracted from dead pupa; O' slide code 3755; GEK leg. et det. deposited MRSN).

\section{Eupithecia laquearia Herrich-Schäffer, 1848}

Eupithecia laquearia Herrich-Schäffer, 1848: pl. 29, figs 181, 182.

MATERIAL EXAMINED. — Larva (L5), 31.VIII.2008, Riserva Naturale Ginepro di Fenecia on Misopates orontium ( $\sigma^{7}$ slide code 3483 GEK leg. et det. deposited MNCM).

NOTES ON FOOD-PLANT. - The finding of a larva on Misopates orontium is a new food-plant record, although Vorbrodt (1928) does cite it on another Scrophulariaceae, Rhiananthus minor.

OTHER OBSERVATIONS. - Not previously documented from the PNAM (Baldizzone et al. 2005).

Subfamily ENNOMINAE Duponchel, 1845

Lomaspilis marginata (Linnaeus, 1758)

Phalaena Geometra marginata Linnaeus, 1758: 527.

Material eXAmined. - Larva (L5), 7.VIII.2010, Sant'Anna di Valdieri, on upper leaf surface of Salix caprea (larva perished; GEK leg. et det. deposited MRSN).

\section{Crocallis elinguaria (Linnaeus, 1758)}

Phalaena Geometra elinguaria Linnaeus, 1758: 520.

Material eXAmined. - Larva (L5), 2.V.2010, Riserva Naturale Ginepro di Fenecia $(861 \mathrm{~m})$ on Acer sp. (Aceraceae) FJGE leg. et

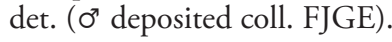

NOTES ON FOOD-PLANT. - Acer (species not known) is not included amongst the food-plants of this moth, although Ebert et al. (2003) list seven plant families amongst those fed upon by the larva.

Other ObSERVATIONS. - Previously cited in the PNAM (Baldizzone et al. 2005).

\section{Colotois pennaria (Linnaeus, 1761)}

Phalaena Geometra pennaria Linnaeus, 1761: 324.

Material eXAmined. - Larva (L4), 2.V.2010, Riserva Naturale Ginepro di Fenecia (895 m) on Crataegus sp. (Rosaceae) (pupa 25.V.2010; deposited coll. FJGE); 11 larvae (L3-L5) 2.V.2010, Riserva Naturale Ginepro di Fenecia $(861 \mathrm{~m})$ on Acer sp. (pupae: 25 \& 30.V.2010; emergences: 14 \& 22.X.2010; 7 \%, 4 o $^{\text {7 deposited }}$ coll. FJGE); 3 larvae (L4), 3.V.2010, Sant'Anna di Valdieri (1022 m) in Crataegus sp. 3 ○ (pupa 27.V.2010; deposited coll. FJGE); larva 
(L4) 4.V.2010, San Lorenzo (880 m) on Prunus spinosa (Rosaceae) (\% emerged 28.X.2010, deposited coll. FJGE); 2 larvae, 5.V.2010, Riserva Naturale Ginepro di Fenecia (911 m), Corylus avellana (L4), Acer sp. (L3) (pupa 27.V.2010) (deposited coll. FJGE); all material FJGE leg. et det.

\section{Phigalia pilosaria}

(Denis \& Schiffermüller, 1775)

Geometra pilosaria Denis \& Schiffermüller, 1775: 100; pl. I a-b, frontispiece.

Material eXAmined. - Larva (L3), 2.V.2010, Riserva Naturale Ginepro di Fenecia (861 m) on Acer sp. (deposited coll. FJGE); FJGE leg. et det.

Notes ON FOOD-PLANT. - Although Ebert \& Steiner (2003) listed eight plant families attacked by this species, Acer is not mentioned as a food-plant.

Other ObSERVATIONS. - Not previously documented from the PNAM (Baldizzone et al. 2005).

\section{Biston strataria (Hufnagel, 1767)}

Phalaena strataria Hufnagel, 1767: 514.

Material EXAminED. — Larva: 1.IX.2008, Riserva Naturale Ginepro di Fenecia (911-927 m): Prunus spinosa (L4) (ơ deposited MNCM); GEK leg. et det.

\section{Biston betularia (Linnaeus, 1758)}

Phalaena Geometra betularia Linnaeus, 1758: 521.

Material EXAMINED. — Larva: 1.IX.2008, Riserva Naturale Ginepro di Fenecia Pyrus communis (L3); larva (L5) 10.VIII.2009, San Lorenzo, Artemisia vulgaris (neither survived); material coll. GEK, leg. et det. GEK.

NOTES ON FOOD-PLANT. — Ebert \& Steiner (2003) mentioned 15 plant families attacked by this species, but their list did not include Pyrus communis, although seven genera of Rosaceae are mentioned.

Other observations. - Previously cited in the PNAM (Baldizzone et al. 2005).

Agriopis aurantiaria (Hübner, 1799)

Geometra aurantiaria Hübner, 1799: pl. 35, fig. 184.

MATERIAl EXAMined. - Larva (L4), 2.V.2010, Riserva Naturale Ginepro di Fenecia (861 m) on Crataegus sp. (perished); coll. FJGE; FJGE leg. et det.

\section{Erannis defoliaria (Clerk, 1759)}

Phalaena defoliaria Clerk, 1759: pl. 7, fig. 4.

Material eXAMined. - 4 larvae (L4), 2.V.2010, Riserva Naturale Ginepro di Fenecia (861 m) (pupated end May 2010; 3 ơ 1 \% emergences: 14.X.2010); two larvae (L4), 3.V.2010, Sant'Anna di
Valdieri (1.022 m) all larvae on Crataegus sp. (\% emerged 15.X.2010) (deposited coll. FJGE) FJGE leg. et det.

Alcis repandata (Linnaeus, 1758)

Phalaena Geometra repandata Linnaeus, 1758: 524.

Material EXAMined. — Larva (L5): 1.V.2010, San Lorenzo (845 m) in Rosa canina ( 9 deposited MNCN) GEK leg. et det.; larva (L4), 3.V.2010, Sant'Anna di Valdieri (924 m) on Corylus avellana (pupa: 25.V.2010; + emerged 14.VI.2010; deposited coll. FJGE) FJGE leg. et det.

\section{Campaea margaritata (Linnaeus, 1767)}

Phalaena Geometra margaritata Linnaeus, 1767: 328.

MATERIAL EXAMINED. — Larva (L5), 3.V.2010, Sant'Anna di Valdieri (1022 m) on Corylus avellana (pupa 8.V.2010 o' emerged 24.V.2010; deposited coll. FJGE) FJGE leg. et det.

NOTES ON FOOD-PLANT. - The larva has not been recorded on Betulaceae, except for Carpinus betulus L. (Ebert \& Steiner 2003).

Other observations. - Previously cited in the PNAM (Baldizzone et al. 2005).

\section{Gnophos furvata meridionalis Wehrli, 1924}

Gnophos meridionalis Wehrli, 1924: 84

Material EXAmined. - Larva (L2) (reared through to L5, when chaetotaxic study was undertaken; see below) 2.IX.2008 under Oxyria digyna growing amongst stones on scree slope (larva perished; in alcohol; deposited MNCN); larva (L4) 4.IV.2009 under Artemisia campestre; larva (L5) taken under Teucrium sp. (Labiatae); larva pupated but pupa perished ( $\%$ genitalia prepared: slide code 3611 GEK leg. et det.; deposited UAM); larvae (L4, L5) 5.IV.2009 taken under Artemisia campestre mat and Helianthemum sp.; larva (L4) 6.IV.2009 taken under Helianthemum sp.; larva (L3) 2.V.2010 taken under Viola sp. growing between rocks; larva (L5) 4.V.2010 on ground between Astragalus and Fumaria officinalis all larvae taken at Riserva Naturale Ginepro di Fenecia (GEK leg. et det.); larva (L5) 3.V.2010, Sant'Anna di Valdieri $(981 \mathrm{~m})$ taken on ground between Lamium (Labiatae) and Rosa; 2 larvae (L5) 4.V.2010, San Lorenzo (845 m) taken under Lathyrus (Leguminosae) and Sedum growing alongside stream (GEK leg. et det.). In captivity these larvae were reared on Rumex crispus and $R$. acetosella L., but only one specimen survived to the pupal stage (see above).

NOTES ON FOOD-PLANT. - Bartsch (2003) mentions the larvae as being hidden amongst low plants in rocky habitats during the day, at ground level, often taken in vicinity of Prunus spinosa (L.) bushes, with another eight plants (in seven families) being cited. This strategy of larvae being hidden was also observed in the study area. If Oxyria digyna were to be confirmed as a food-plant, this would be a new record; polygonales do not figure amongst plants attacked, although these are accepted in captivity (see above).

Other observations. - Previously cited in the PNAM (Baldizzone et al. 2005). 


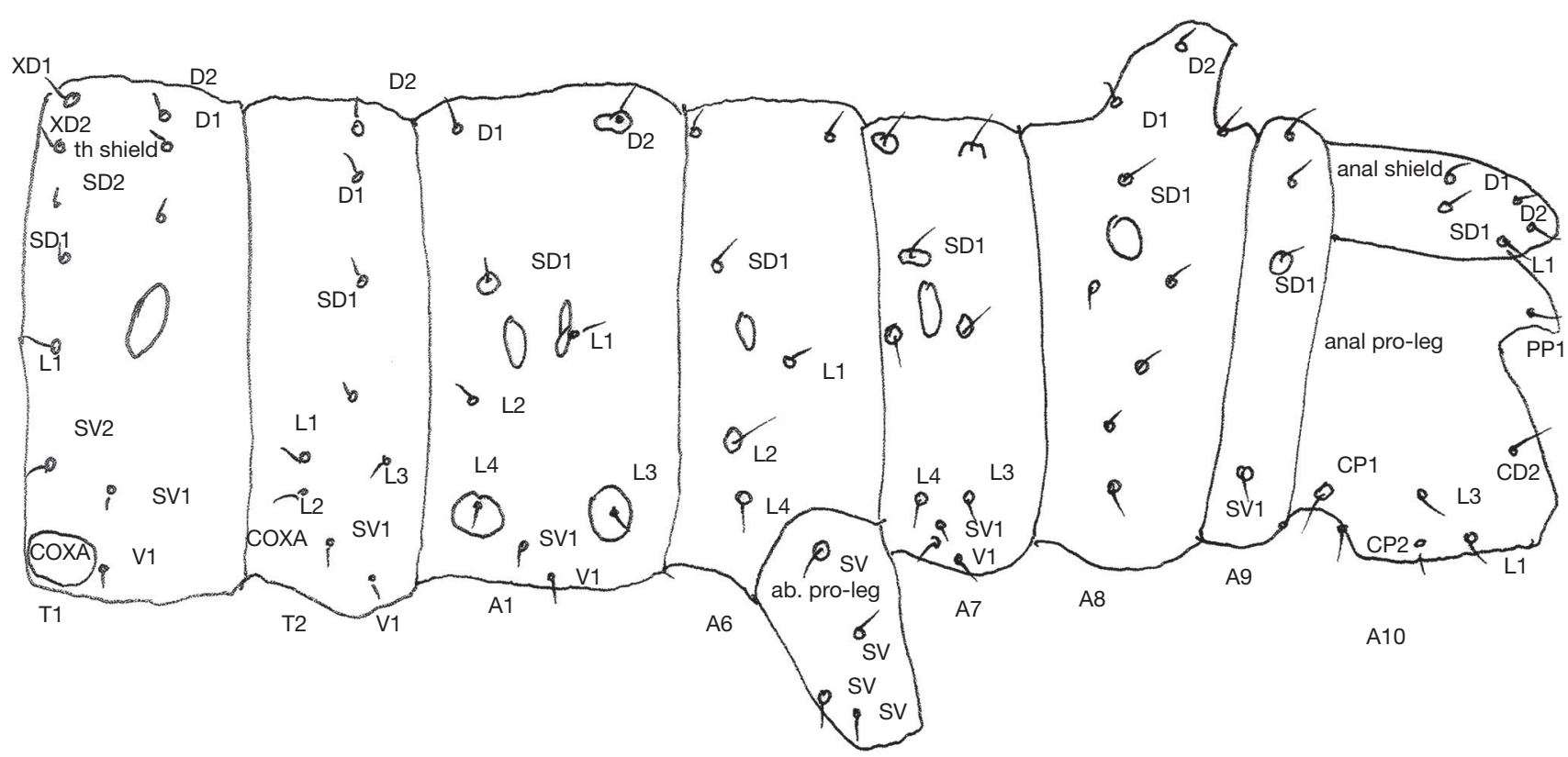

FIG. 1. - Gnophos furvata meridionalis Wehrli, 1924: chaetotaxy: L5: 2.VIII.2008 (Valdieri). Abbreviations: see text. Specimen length: 22 mm (preserved specimen).

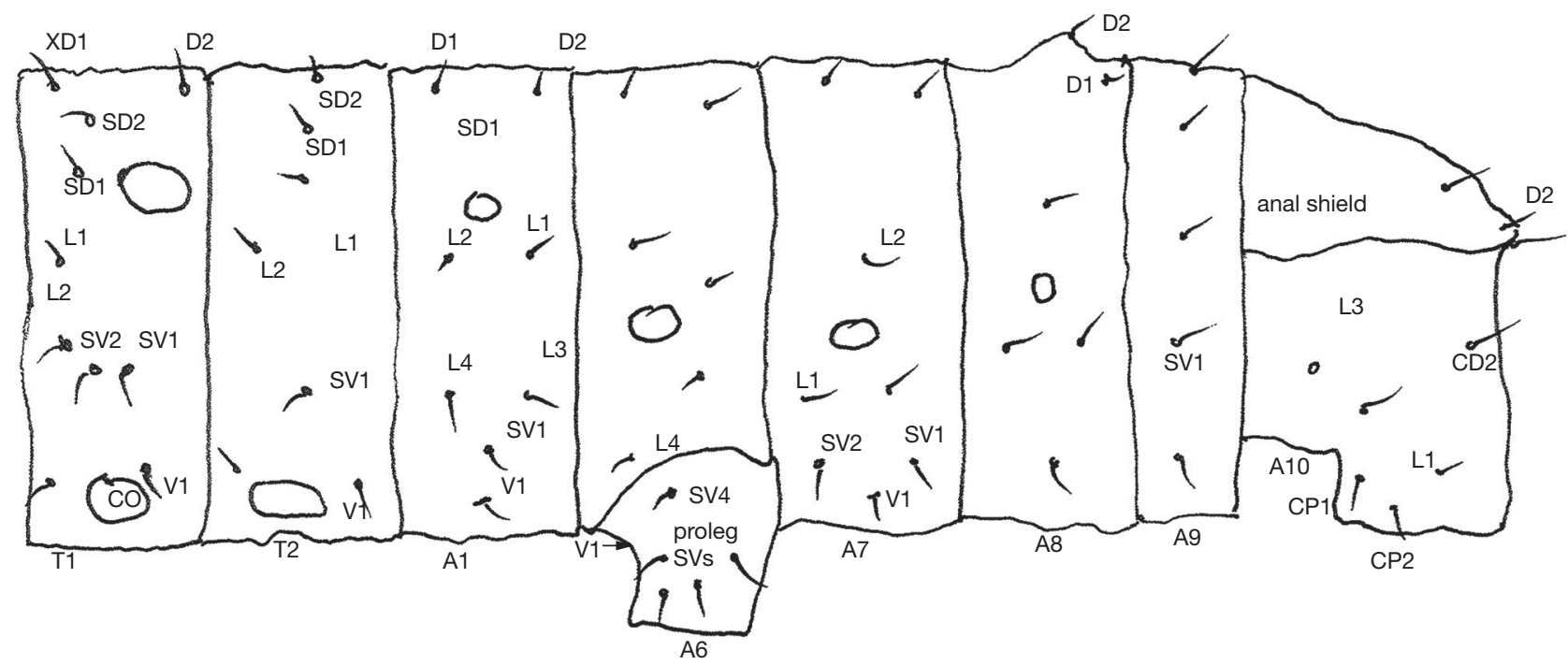

FIG. 2. - Charissa pullata ([Denis \& Schiffermüller], 1775): chaetotaxy: L5: V.11 (Sant'Ana di Valdieri). Abbreviations: see text. Specimen length: 18.5 mm.

\section{LARVAL DESCRIPTION}

L5 (22 mm; preserved specimen) ( $\mathrm{n}=1)$ : (Fig. 1) dark ochre dorsal zone, lighter ochre ventral and lateral zones; setae short; protuberance in A8 dorsal zone which carries D2 setae; T1 roughly triangular-shaped, dark ochre from spiracle to T2; similar pattern repeated on T3, also A1 near spiracle, but much smaller; A10 abdominal pro-legs dark ochre in reduced area of anterior zone near PP1 setae. Chaetotaxy of L5 (Fig. 1): T1 with thoracic shield indistinct; all setae very short; D2, D1, with latter just below former; SD1, SD2 (latter about 50\% length of former); lateral setae (L1-L3) in anterior zone of segment, well before spiracle; SV1, SV2, anterior to coxa; V1; T2: D2, D1, SD1 (SD2 not discernible); L1, L2, L3 distant from the first two setae; SV1 unisetose; V1; A1: D1, D2 (dark ochre pinnacle surrounded by lighter ochre patch); A6: D1, D2; SD1, L1, L2 in vicinity of spiracle with L2 twice as far from this structure than are the other lateral setae; L4 with four SV setae on anterior surface of A6 proleg.; V1 on inside of proleg.; A7: D1, D2; SD1, L1, L2 close to spiracle; inferior lateral L4, L3 at same level on urite; SV1, SV2, then V1 ventrally; A8: pair of protuberances dorsally with D2 uppermost and then D1; A9: setae in single descending row, beginning with $\mathrm{D}$ setae, with SD1, SV1 relatively prominent, being c. 20\% longer than the other setae; A10: anal shield somewhat wedge-shaped, protruding slightly over anal proleg.; D2 c. 10\% longer than PP1 (anterior zone of anal proleg.); CD2 almost level with CP1. 


\section{Charissa pullata \\ ([Denis \& Schiffermüller], 1775)}

Geometra pullata Denis \& Schiffermüller, 1775: 108.

Material eXAmined. - 4 larvae (L5), 28.V.2011, Ubac di Rugeral $(1319 \mathrm{~m})$ (emerged ơ deposited MRSN; larval specimens deposited UAM); two larvae (L5), 29.V.2011, Lago della Rovina (1556-1559 m) (deposited coll. UAM) all larvae on ground under mats of Rumex scutatus; GEK leg. et det.

NotES ON FOOD-PLANT. - All six larvae taken in May 2011 were found on the ground under mats of Rumex scutatus. As is with the case with larvae of $G$. $f$. meridionalis, this species has not been taken in the wild associated with the family Polygonaceae (Bartsch 2003), although its larva fed on this plant family in captivity.

Other observations. - Previously cited in the PNAM (Baldizzone et al. 2005).

\section{LARVAL DESCRIPTION}

L5 (18.5 mm; preserved specimens) $(\mathrm{n}=2)$ (Fig. 2$)$ : dorsal zone light ochre, dorsal line slightly darker ochre; lateral zones cocoa-ochre with lower lateral areas same shade as dorsal area, ventrally cocoa-ochre; setae short, golden ochre; A8 with dorsal protuberance carrying D2 setae. Chaetotaxy: L5: T1: XD1, XD2 in thoracic shield; D1, D2; SD1 below SD2 the former $c .50 \%$ longer than the latter; L1, L2 very close together on cuticular ridge; SV1, SV2, V1; T2: D1, D2, SD2 thicker and c. 50\% length of SD1; L1, L2 almost at same level on pronounced cuticular ridge; SV1 relatively pronounced, V1; A1: D1, D2; SD1 (SD2 not visible), L1 and L2 form "triangle" around spiracle; L4, L3 immediately below the aforementioned setae almost level with each other; SV1, V1; A6: L4 on raised "ridge", SV4 on abdominal pro-leg, these two setae both "thicker" more wedge-shaped and 50\% shorter than remaining four SV setae on pro-leg surface, which are relatively long; V1 same length on inferior surface of pro-leg placed centrally; A7: setae wedge-shaped, L1, L2, L3 (in vicinity of spiracle) are $c .60 \%$ shorter than setae of previous urite; SV1, SV2, V1; A8: protuberance dorsally with D2, D1 seta below; A9: setae in descending row from D setae, with SV1 the most prominent (c. 10\% longer); A10: relatively prominent wedge-shaped anal shield: CD2 anterior to $\mathrm{CP} 1$; L3 relatively prominent in anterior zone of anal pro-leg; CP1, CP2 and L1 c. 10\% longer than other setae, slightly curved at apex.

\section{Perconia strigillaria (Hübner, 1787)}

Phalaena strigillaria Hübner, 1787: 13, pl. 2, fig. I (fig. F).

MATERIAL EXAMINED. — Larva: 4.IV.2009, Riserva Naturale Ginepro di Fenecia (911-927 m) underneath mat of Artemisia campestre, though without establishing this as the food-plant (larva did not survive) (GEK leg. et det. material not kept).

\section{DISCUSSION}

The Larentiinae are regarded as being typical of high altitude areas (Kaila et al. 1996; Brehm \& Fiedler 2003; Baldizzone et al. 2005), in contrast, the Sterrhinae are not well represented at high altitudes (Kaila et al. 1996; Hausmann 2004; Bodner et al. 2009). In the present study, the Larentiinae were represented by 13 larvae (12.6\%) of seven species (25\%), whereas the Sterrhinae were better represented by 26 larvae (25.2\%) of eight species $(28.6 \%)$, suggesting that the methodology chosen to collect the larvae could explain this apparent anomaly. Although the Ennominae as a subfamily were found to be the best-represented taxon (13 taxa $=46.4 \%$; 66 larvae $=64.1 \%$ ), three taxa (Charissa pullata, Colotois pennaria and Gnophos furvata meridionalis) accounted for 34 larvae (33\%), of which $17(16.5 \%)$ - belonging to the two taxa of Gnophini - are ground dwellers (Bartsch 2003). Strikingly, the Eupitheciini Pierce, 1914, which are faunistically important in the Italian Maritime Alps, with 29 species $(=24.8 \%$ of the Larentiinae) according to Baldizzone et al. (2005), were only represented in the present study by one species $(3.6 \%)$, namely E. laquearia.

\section{MORPHOLOGICAL CONSIDERATIONS CONCERNING LARVAE OF GNOPHINI}

There have been no chaetotaxic studies undertaken on any larvae in this ennomine tribe, although the larvae of Gnophos furvata furvata ([Denis \& Schiffermüller], 1775) and C. pullata are illustrated in Bartsch (2003), in the present paper we have provided details on chaetotaxy of these two taxa, although the small size of the samples studied only allows tentative conclusions. Both larvae of these two taxa show a pair of protuberances in the dorsal area of A8 which carry the D2 seta, this feature can also be seen in larvae of Euchrognophos mucidarius (Hübner, 1799) (King unpubl. obs.) and in some larvae of the genus Dyscia Hübner, [1825] (Trusch \& Erlacher 2001) (Gnophini), but it has also been seen in C. pennaria (Colotoini Guenée, 1845) (in which species it is vermillion) (Soria 1987) and commented on in other ennomine larvae (Bocaz et al. 2003). However, it does not occur in the related Aspitates ochrearia (Rossi, 1794) (Gnophini) (anal shield A10 bifurcate), so it cannot be regarded as a tribal synapomorphy. In the Nearctic species Lytrosis permagnaria Packard, 1948, cuticular processes are noted on A1 and A5, rather than on A8 (Wagner et al. 2003). Young (2008) noted the extraordinary number of SV setae on the anterior face of the abdominal proleg (A6), which in the two taxa described in this paper varies between four (G.f. meridionalis) and five (C. pullata). An additional factor to be taken into account is that of individual larval variation, which would require considerable sampling of the taxa under consideration (Mutanen et al. 2009). Because this paper has only dealt with a small sample of L5 larvae of only two species in a tribe as speciose as Gnophini (Ebert et al. 2003; Redondo et al. 2009), more material would be needed $a b$ ovo in order to examine phylogenetic relationships between the taxa. 


\section{Acknowledgements}

The All Taxa Biodiversity Inventory + Monitoring Mercantour/ Alpi Marittime was launched by the European Distributed Institute of Taxonomy (EDIT) project (2006-2011). The ATBI was coordinated by M.-F. Leccia (Parc national du Mercantour) and M. De Biaggi (Parco Naturale Alpi Marittime), who provided the relevant permits. EDIT contributed towards the cost of travel and accommodation on the seven trips undertaken, as did the Parco Naturale Alpi Marittime, which subsidized the trips undertaken in 2011 (Alcotra 20072013 and its Generalized Biological Inventory programme).

We are grateful to Mark Shaw (Royal Museum of Scotland, Edinburgh) for having identified the hymenopteran parasitoids mentioned in this paper, to David Wagner at the Department of Ecology \& Evolutionary Biology, University of Connecticut, USA, who helped the first author in the field of chaetotaxy and who made his stay there (November 2011 to January 2012) so very memorable, and the Biology Department of the Autónoma University, Madrid, for all facilities provided and the granting of a three-month's subsidy in order to be able to travel to the University of Connecticut. We also add our most grateful thanks to Sven Erlacher and to an anonymous reviewer comments from whom improved our paper.

\section{REFERENCES}

Ahola M. \& SiLVONEN K. 2005. - Larvae of Northern European Noctuidae. Vol. I. Kuva Seppälä Yhtiöt Oy, Helsinki, 657 p.

AsCHMANN H. 1984. - A restrictive definition of Mediterranean climates. Bulletin de la Société botanique de France 131 (2-4): 21-30.

Baldizzone G., Fiumi G. \& Gianti M. 2005. — Geometridae del Parco Naturale Alpi Marittime (Piemonte, Italia) (Lepidoptera). Bollettino di Museo Regionale di Scienze Naturali Torino 22 (2): 443-516.

BARBERO M. 1979. - Les remontées méditerranéennes sur le versant italien des Alpes. Écologie Mediterranea 4: 109-132.

BARBERO M. 1981. - Les pénétrations méditerranéennes dans la haute montagne alpienne. Anales del Jardín Botánico de Madrid 37 (2): 301-314.

BARbero M. G., Bonin \& P. QUÉZEI. 1971. — Signifícation bioclimatique des pelouses écorchées sur les montagnes du pourtour méditerranéen; leurs relations avec les forêts d'altitude. Bulletin de la Société botanique de France 118 (suppl. 2): 17-56.

BarTsCH D. 2003. - Die Steinspanner der Gattungen Gnophos, Charissa und Elophos, in EBERT G. (ed.) Die Schmetterlinge BadenWürttembergs 9: 548-568.

BECK H. 1960. - Die Larvalsystematik der Eulen (Noctuidae). Abhandlung Larvalsystematik Inseckten 4. Akademie-Verlag, Berlin, 406 p.

BlaNCHARD R. 1956. - Les Alpes occidentales, vol. 7, Essai d'une Synthèse. Arthaud, Grenoble, 605 p.

Bocaz P., PARRa L. E. \& Victoriano P. F. 2003. — Larval morphological variation and its relation to host plants in Syncirsodes primata (Lepidoptera: Geometridae). Gayana 67 (1): 39-44.

Bodner F., Brehm G., Homeier J., Strutzenberger P. \& Fiedler F. 2009. - Caterpillars and host plant records for 59 species of Geometridae (Lepidoptera) from a montane rainforest in southern Ecuador. Journal of Insect Science 10 (67): 1-22.

BREHM G. \& FiEDLER K. 2003. — Faunal composition of geometrid moths changes with altitude in an Andean montane rain forest. Journal of Biogeography 30: 431-440.
Carrara G. 1926-1928. - I Macrolepidotteri del territorio di Trieste, in appendices: Elenco dei Macro lepidotteri raccoti nel Territorio di Trieste da F. A. Vogel. Atti del Museo Civico di Storia Naturale Trieste 11: 63-116.

ClerCK C. 1759. - Icones Insectorum rariorum Cum Nominibus eorum trivialibus, locisqve e C. Linnei Arch. R. et Eqv. Aur. Syst. Nat. allegatis. Sectio Prima. Holmiae, [8 unnumbered pages] pl. 1-16.

Covell C. V. 1983. - The state of our knowledge of the Neotropical Sterrhinae (Geometridae). Second Symposium on Neotropical Lepidoptera, Arequipa, Peru 1983, Supplement 1: 17-23.

[DENIS M. \& SCHIFFERMÜLlER J. I.] 1775. — Ankündung eines systematischen Werkes von den Schmetterlingen der Wienergegend herausgegeben von einigen Lehrern am $k$. $k$. Theresianum. Wien (Augustin Bernardi), 323 p., pl. I, frontispiz.

DUGDALE J. S. 1961. — Larval characters of taxonomic significance of New Zealand Ennomines (Lepidoptera: Geometridae). Transactions of the Royal Society of New Zealand 16: 215-233.

EBERT G. \& STEINER A. 2001a. - Sterrhinae in Die Schmetterlinge Baden-Wüttermbergs, Band 8: Nachfalter VI, Geometridae, Verlag E. Ulmer, Stuttgart, 541 p.

Ebert G. \& STEINER A. 2001b. - Larentiinae in Die Schmetterlinge Baden-Wüttermbergs, Band 8: Nachfalter VI, Geometridae, Verlag E. Ulmer, Stuttgart, 541 p.

Ebert G., Steiner A. \& Trusch R. 2003. - Ennominae in Die Schmetterlinge Baden-Wüttermbergs, Band 9: Nachfalter VII, Geometridae, Verlag E. Ulmer, Stuttgart, 609 p.

Eitschberger U., ReinHARDT R. \& STEINIGER H. 1991. — Wanderfalter in Europa (Lepidoptera). Atalanta 22 (1): 1-67.

Falcó J. V., Moreno J. \& Jiménez R. 1997. — Datos sobre Ciclostominos españoles: III. Rogadinae s. str. (Hymenoptera: Braconidae). Boletín de la Asociación Española de Entomología 21: 1-2: 55-64.

Fielding C. A. \& Coulson J. C. - 1995. A test of the validity of insect food-plant and life-history records: Lepidoptera on heather (Calluna vulgaris). Ecological Entomology 20: 343-347.

Flamigni C., FiUMI G. \& PARENZAN P. 2007. — Lepidotteri eteroceri d'Italia. Geometridae Ennominae I. Natura Edizioni Scientifiche, Bologna, $384 \mathrm{p}$.

FORBES W. T. M. 1910. — A structural study of some caterpillars. Annals of the Entomological Society of America 3: 94.

Gallé L., Margóczi L., KovÁcs E., GYÖRfFy G., KÖMÖRCZI L. \& NeMETH L. 1995. — River valleys: are they ecological corridors? Tiscia 29: 53-58.

Goeze J. A. E. 1781. — Entomologische Beyträge zu des Ritter Linné zwölften Ausgabe des Natursystems, 3. Leipzig (Weidmanns Erben und Reich). Teil, 3. Band. I-XLVIII + 1-439.

Gómez de Aizpúrua C., González Granados J. \& Viejo MonTESINOS J. L. 2006. - Mariposas y sus biotopos, Lepidoptera III memoria 2004. Reserva Natural El Regajal-mar de Ontígola, Madrid, $299 \mathrm{p}$.

HASSENFUSS I. 1963. - Eine vergleichend-morfologische Analyse der regulären Borstenmuster der Lepidopterenlarven. Zeitschrift für Morphologie und Ökologie der Tiere 52: 197-364.

Hausmann A. 2004. - Sterrhinae, in Hausmann A. (ed.): The Geometrid Moths of Europe, 2. Apollo Books, Stenstrup, 600 p.

Heitzman R. L. 1973. - Life history studies of Idaea obfusaria (Walker) (Geometridae). Journal of Research on the Lepidoptera 12 (3): 145-150.

HERRICH-SCHÄFFER G. A. W. (“1847”) [1843-1855]. — Systematische Bearbeitung der Schmetterlinge von Europa, zugleich als Text, Revision und Supplement zu Jakob Hübner's Sammlung europäischer Schmetterlinge, Dritter Band. Die Spanner. G. J. Manz, Regensburg, $184+34$ p., 90 pls.

HiNTON H. E. 1946. - On the homology and nomenclature of the setae of lepidopterous larvae, with some notes on the phylogeny of the Lepidoptera. Transactions of the Entomological Society of London 97: 1-37. 
HÜBNER J. 1787. - Beiträge zur Geschichte der Schmetterlinge. Zweiter Theil. Published by the author, Augsburg, 29 p., 4 pl.

HÜBNER J. [1796-1838]. — Sammlung europäischer Schmetterlinge. Vol. 5: Geometrae. Published by the author, Augsburg, 24 p, 113 pls.

Huemer P. 1998. — Endemische Schmetterlinge der Alpen - ein Überlick (Lepidoptera). Stapfia 55: 229-256.

Huemer P. \& Morandini C. 2006. - Chesias angeri Schawerda, 1919 stat. rev., a long neglected species from northern Italy. Nota lepidopterologica 28 (3/4): 167-175.

HufNAGEL J. S. 1767. - Fortsetzung der Tabelle von den Nachtvoegeln, welche die 3e Art derselben, nehmlich die Spannenmesser (Phalaenas Geometras Linnaei) enthält. Berlinisches Magazin 4 (6): 599-626.

Kaila L., Viidalepp J., MikKola K. \& Mironov V. 1996. — Geometridae (Lepidoptera) from the Tian-Shan Montains in Kazakhstan and Kyrgyzstan, with descriptions of three new species and one new subspecies. Acta Zoologica Fennica 200: 57-82.

King G. E. \& Viejo MonTesinos J. L. 2010. — Geometrid larvae and their food-plants in the South of Madrid (Central Spain) (Geometridae). Nota lepidopterologica 33 (1): 45-61.

King G. E. \& Viejo Montesinos J. L. 2012. — Las larvas del género Idaea Treitschke, 1825 (Insecta: Lepidoptera: Geometridae), nuevos datos sobre su morfología y biología. Graellsia 68 (2): 249-262.

LINNAEUS C. 1758. - Systema naturae per regna tria naturae, secundum classes, ordines, genera, species, cum characteribus, differentiis, synonymis, locis. Tomus I. Editio decima, reformata. Laurentius Salvius, Holmiae, 824 p.

LinNaEus C. 1761. - Fauna Svecica Sistens Animalia Sveciae Regni: Mammalia, Aves, Amphibia, Pisces, Insecta, Vermes. Distributa Per Classes \& Ordines, Genera \& Species, Cum Differentiis Specierum, Synonymis Auctorum, Nominibus Incolarum, Locis Natalium, Descriptionibus Insectorum. Editio Altera, Auctior. Salvius, Stockholm, $578 \mathrm{p}$.

LinNaEus C. 1767. - Systema Naturae, Tom. I. Pars. II, Editio duodecima reformata. Salvius, Holmiae: 533-1327 + unpaginated supplement.

MÜller O. F. 1764. - Fauna Insectorum Fridrichsdalina, sive methodica descriptio insectorum agri Fridrichsdalensis, cum characteribus genericis et specificis, nominibus trivialibus, locis natalibus, iconibus allegatis, novisque pluribus speciebus additis. I-XXIV. Frid. Gleditschii, Hafniae et Lipsiae, 96 p.

Mutanen M., RuOTSAlainen H. \& Kaila L. 2009. — Variation in larval chaetotaxy in Orthosia gothica, effects of mother, sex and side, and implications for systematics. Systematic Entomology 34 (4): 712-723.

OzENDA P. 1966. - Perspectives nouvelles pour l'étude phytogéographique des Alpes du Sud. Documents pour la carte de la végétation des Alpes 4: 1-198.

PARENZAN P. \& PORCELLI F. 2006. - I macrolepidotteri italiani Fauna Lepidopterorum Italiae (Macrolepidoptera). Phytophaga 15: 1-1051.

Redondo V. M., Gastón F. J. \& Gimeno R. 2009. — Geometridae Ibericae. Apollo Books, Stenstrup, $361 \mathrm{p}$.

Ruohomäki K., Tanhuanpä́a M., Ayres M. P., Kaitaniemi P., TAMmaru T. \& Haukioja E. 2000. - Causes of cyclicity of Epirrita autumnata (Lepidoptera, Geometridae): grandiose theory and tedious practice. Population Ecology 42: 211-223.

SANNINO L. \& ESPINOSA B. 1999. — Aspetti morfologici ed etiologici di Scopula turbidaria (Lepidoptera, Geometridae). Fragmenta entomologica 31 (2): 377-395.
SANNinO L. \& EsPinOSA B. 2002. - Note sulla morfología degli stadi preimmaginali di Idaea seriata (Lepidoptera, Geometridae). Fragmenta entomologica 31 (2): 189-206.

SANNINO L., EsPinOSA B. \& BALBIANI A. 1995. - Morfologia comparativa negli stadi preimmaginali di lepidotteri del tabacco 2. Larve: Bolletino del Laboratorio di Entomologia Agraria, 'Filippo Silvestri' 50 (1993): 23-91.

SATO R. 1984. - Taxonomic study of the genus Hypomecis Hübner and its allied genera from Japan (Lepidoptera: Geometridae: Ennominae). Special Bulletin Essa Entomological Society 1: 1-213.

SCOPOLI J. A. 1763. - Entomologia Carniolica exhibens insecta Carnioliae indigena et distributa in ordines, genera, species, varietates. Methodo Linnaeana, Trattner, Vindobonae, $421 \mathrm{p}$.

SHAw M. R. 1997. — Rearing parasitic Hymenoptera. The Amateur Entomologist 25: 1-52.

Shaw M. R. \& Huddleston T. 1991. - Classification and Biology of Braconid wasps. Handbooks for the Identification of British Insects 7 (11): 1-126.

SINGH B. 1951. - Immature stages of Indian Lepidoptera, No. 8: Geometridae. Indian Forest Records 8 (7): 67-160.

SORIA S. 1987. - Lepidópteros defoliadores de Quercus pirenaica Willdenow, 1805. Fuera de serie ${ }^{\circ} 7$, Ministerio de Agricultura, Pesca y Alimentación, Madrid, 302 p.

STEHR F. W. (ed.) 1987. - Immature Insects. Volume 1. Kendall/ Hunt Publishing Company, Dubuque, Iowa, 754 p.

TRusCh R. \& ERLaCher S. 2001. - Zur Morphologie, Verbreitung, Bionomie und Identifikation der Dyscia-Arten (Lepidoptera: Geometridae; Ennominae). Bonner Zoologische Monographien 49: $1-115$.

Turlings T. C. J., Wäckers F. L., Vet L. E. M., LeWIS W. J. \& TuMLINSON J. H. 1993. - Learning of host-finding cues by hymenopterous parasitoids, in PAPAJ D. J. \& LEWIS A. C. (eds), Insect Learning, Ecological and Evolutionary Perspectives. Klewer Academic Publishers, New York, 51-78.

Villemant C., Daugeron C., Gargominy O., Isaia M., DeharVENG L. \& JUDSON M. L. I. 2015. - The Mercantour/Alpi Marittime All Taxa Biodiversity Inventory (ATBI): achievements and prospects, in DAUGERON C., DeHARVENG L., ISAIA M., VILLEMANT C. \& JUDSON M. (eds), Mercantour/Alpi Marittime All Taxa Biodiversity Inventory. Zoosystema 37 (4): 667-679. http:// dx.doi.org/10.5252/z2015n4a10 Vorbrodt K. 1928. — Die Schmetterlinge von Zermatt. Deutsche entomologishe Zeitschrift Iris $42(1 / 2): 7-130$.

Wagner D. L., Hosler E. W. \& Hosler F. E. 2003. - The larva and pupa of Lytrosis permagnaria Packard (Geometridae). Journal of the Lepidopterists' Society 57 (2): 107-112.

WARING P. 2004. - The Bright Wave Moth Idaea ochrata (Scop.) (Lepidoptera: Geometridae) captive-reared for five generations solely on Hare's-foot Clover Trifolium arvense L. and Common Chickweed Stellaria media, with minimal intervention. Entomologist's Record and Journal of Variation 116: 138-139.

Wehrli E. 1924. — Nice-St.-Martin-Vésubie-Digne: Ein Beitrag zur Lepidopterenfauna der Alpes Maritimes und der Basses Alpes. Deutsche Zeitschrift Iris 38: 59-98.

WINTER W. D. 2000. - Basic Techniques for Observing and Studying Moths and Butterflies. The Lepidopterists' Society Memoir 5444 p. Young C. J. 2008. - Characterization of the larvae of the Australian Nacophorini. Zootaxa 1862: 1-74. 


\section{APPENDIX 1}

Plants (to species level) mentioned in the present study (includes literature records).

Papaveraceae Juss.

Fumaria officinalis L.

Cupressaceae Gray

Juniperus phoenica L.

Betulaceae Gray

Betula alba L.

Corylus avellana $\mathrm{L}$.

Salicaceae Mirb.

Salix caprea L.

Polygonaceae Juss.

Rumex crispus $\mathrm{L}$.

Rumex conglomeratus Murray

Rumex acetosella $\mathrm{L}$
Oxyria digyna L. (Hill.)

Rosaceae Juss.

Rosa canina L.

Prunus spinosa $\mathrm{L}$.

Pyrus communis $\mathrm{L}$.

Amelanchia ovalis Medik.

Scrophulariaceae Juss.

Misopates orontium L. Raf.

Rhiananthus minor $\mathrm{L}$.

Rubiaceae Juss.

Galium sylvaticum L.

Asteraceae Bercht. \& J.Presl

Artemisia vulgaris L. 